\title{
ON PERIODICITY OF ENTIRE FUNCTIONS
}

\author{
CHUNG-CHUN YANG
}

AbStract. A sequence $S=\left\{s_{n}\right\}$ is said to be a periodic set of period $\tau(\neq 0)$ if and only if $S^{*}=\left\{s_{n}+\tau\right\}$ can be rearranged to be a sequence to coincide with $S$. Let $F$ be the class of all entire functions $f$ satisfying the growth condition:

$$
\limsup _{r \rightarrow \infty} \log \log \log M(r, f) / \log r<1 .
$$

In this paper it is shown that if $f \in F$ and the zero sets of $f$ and $f^{\prime}$ both are periodic sets with the same period $\tau$, then $f$ can be expressed as $f(z)=e^{c z} g(z)$, where $c$ is a constant and $g(z)$ is a periodic entire function with period $\tau$. A counterexample is exhibited to show that the above condition is a necessary one.

1. Introduction. Let $S=\left\{s_{n}\right\}$ denote a sequence of complex numbers. $S$ is called a periodic set with period $\tau(\neq 0)$ iff $S^{*}=\left\{s_{n}+\tau\right\}$ can be rearranged to be a sequence to coincide with $S$. Accordingly, an empty set is a periodic set. Let $f$ denote an entire function and $Z_{f(z)}$ denote the zero set of $f(z)$. Clearly, if $f$ is a periodic function with period $\tau$, then all the sets $Z_{f^{(n)}(z)}, n=0,1,2, \cdots$, are periodic sets with the same period $\tau$. However, the converse of the above result is not true. That is, if all the zero sets $Z_{f(n)}, n=0,1,2, \cdots$, of an entire function $f$ are periodic sets with the same period $\tau$, it does not follow that $f$ is an entire function with period $\tau$. To verify this, choose any complex number $b \neq$ rational number and $f(z)=e^{i b z} \sin z$. Then it is easy to see that all the zero sets $Z_{f^{(n)}(z)}, n=0,1,2, \cdots$, are periodic sets with the same period but that $f(z)$ fails to be a periodic function. More generally, for any constant $c$ and periodic entire function $g(z)$, the function $f$ of the form

$$
f(z)=e^{c z} g(z)
$$

has the property that all the zero sets $Z_{f^{(n)}(z)}, n=0,1,2, \cdots$, are periodic sets with the same period.

It becomes natural for us to ask the following question: Does form (1) include all entire functions $f$ such that all zero sets $Z_{f^{(n)}(z)}, n=0,1,2, \cdots$, are periodic sets with the same period?

Received by the editors April 30, 1973 and, in revised form, August 20, 1973.

AMS (MOS) subject classifications (1970). Primary 30A64, 30A66. 
We are unable to answer this question completely. However, if one puts a certain growth restriction on the functions to be considered, then a stronger result can be obtained as follows:

THEOREM. Let $f$ be an entire function with

$$
\underset{r \rightarrow \infty}{\limsup } \log \log \log M(r, f) / \log r<1 .
$$

Then $f$ has the form (1) if and only if the zero sets $Z_{f(z)}$ and $Z_{f^{\prime}(z)}$ are periodic sets with the same period $\tau$.

Remarks. (A) The Theorem is true for any entire function of finite order.

(B) The Theorem may be false if condition (2) is violated. There is no difficulty in showing that $f(z)=\exp \left(e^{z / 2}-e^{z / \sqrt{ } 2}\right)$ is a counterexample. We omit the verification here.

Proof. The necessity is obvious. We proceed to the sufficiency. By assumption, we have, for some complex number $\tau \neq 0$,

$$
f(z) / f(z+\tau)=e^{\alpha(z)},
$$

and

$$
f^{\prime}(z) / f^{\prime}(z+\tau)=e^{\beta(z)},
$$

where $\alpha(z)$ and $\beta(z)$ are entire functions. We will now show that $\alpha^{\prime}$ is identically zero. Suppose that $\alpha^{\prime}(z) \not \equiv 0$. Then from equation (3) we have

$$
f(z)=f(z+\tau) e^{\alpha(z)},
$$

and hence

$$
f^{\prime}(z)=f^{\prime}(z+\tau) e^{\alpha(z)}+\alpha^{\prime}(z) f(z+\tau) e^{\alpha(z)} .
$$

Thus combining equations (5) and (6) and using equation (4), equation (6) becomes

$$
f^{\prime}(z+\tau)\left[e^{\beta(z)}-e^{\alpha(z)}\right]=\alpha^{\prime}(z) f(z+\tau) e^{\alpha(z)} .
$$

Then replacing $z$ by $z+\tau$ in equation (7),

$$
f^{\prime}(z+2 \tau)\left[e^{\beta(z+\tau)}-e^{\alpha(z+\tau)}\right]=\alpha^{\prime}(z+\tau) f(z+2 \tau) e^{\alpha(z+\tau)} .
$$

Now assume that $e^{\beta(z+\tau)}-e^{\alpha(z+\tau)} \neq 0$; otherwise $\alpha^{\prime}(z+\tau) \equiv 0$, giving a contradiction. Thus

$$
\frac{f^{\prime}(z+\tau)}{f^{\prime}(z+2 \tau)} \frac{e^{\beta(z)}-e^{\alpha(z)}}{e^{\beta(z+\tau)}-e^{\alpha(z+\tau)}}=\frac{\alpha^{\prime}(z)}{\alpha^{\prime}(z+\tau)} \frac{f(z+\tau)}{f(z+2 \tau)} e^{\alpha(z)-\alpha(z+\tau)} .
$$


By substituting equations (3) and (4) into equation (9),

$$
e^{\beta(z+\tau)} \frac{e^{\beta(z)}-e^{\alpha(z)}}{e^{\beta(z+\tau)}-e^{\alpha(z+\tau)}}=\frac{\alpha^{\prime}(z)}{\alpha^{\prime}(z+\tau)} e^{\alpha(z)} .
$$

We set $\alpha^{\prime}(z) / \alpha^{\prime}(z+\tau)=h(z)$ and so deduce

$$
e^{\beta(z)}-e^{\alpha(z)}=h(z) e^{\alpha(z)-\beta(z+\tau)}\left(e^{\beta(z+\tau)}-e^{\alpha(z+\tau)}\right),
$$

and hence

$$
1+h(z)=e^{\beta(z)-\alpha(z)}+h(z) e^{\alpha(z+\tau)-\beta(z+\tau)} .
$$

To finish the proof, we need to estimate the growth of $h(z)$. We first recall (cf. [2, p. 216]), that the order of the product or the quotient of two meromorphic functions $f_{1}$ and $f_{2}$ of order $\lambda_{1}$ and $\lambda_{2}$ respectively is $\leqq \max \left(\lambda_{1}, \lambda_{2}\right)$, and equality holds provided $\lambda_{1} \neq \lambda_{2}$. We assert now that the order of $h$ must be less than 1 , since otherwise either the order of $\alpha^{\prime}(z)$ (hence $\alpha(z)$ ) or that of $\alpha^{\prime}(z+\tau)$ (hence $\alpha(z+\tau)$ ) must be greater than or equal to 1 . It then follows from equation (3) that the function $f(z) / f(z+\tau)$ grows at least as fast as $\exp e^{r^{1-\varepsilon}}$ for any given positive small number $\varepsilon>0$. This implies that either the function $f(z)$ (hence $f(z+\tau)$ ) or the function $f(z+\tau)$ (hence $f(z)$ ) grows at least as fast as $\exp e^{r^{1-\varepsilon}}$. This will contradict condition (2) by choosing $\varepsilon$ sufficiently small. Now we consider two cases: (i) $\beta(z)-\alpha(z)$ is a nonconstant entire function, and (ii) $\beta(z)-\alpha(z)$ is a constant. In case (i) we have from equation (12) that

$$
\delta\left(e^{\beta(z)-\alpha(z)}, 0\right)=\delta\left(e^{\alpha(z+\tau)-\beta(z+\tau)} h-(1+h(z)), 0\right)=1,
$$

where $\delta(f, a)$ denotes the Nevanlinna deficiency for the function $f$ at the value $a$. For a standard reference for this notion see [1].

By noting that $\delta\left(e^{\alpha(z+\tau)-\beta(z+\tau)} h, 0\right)=1$ and using the analog result of Nevanlinna's second fundamental theorem for deficient functions [1, p. 47], we have to conclude that $1+h(z) \equiv 0$; otherwise, $e^{\alpha(z+\tau)-\beta(z+\tau)} h$ would have three deficient functions: $0,1+h$, and $\infty$ of slower order of deficiency 1. It follows that $\alpha^{\prime}(z) \equiv-\alpha^{\prime}(z+\tau)$, since $\alpha^{\prime}(z) / \alpha^{\prime}(z+\tau) \equiv-1$. Hence $\alpha^{\prime}(z)$ has an order at least 1 which will lead to a contradiction, as we analysed before.

In case (ii) we have from equation (12) again that

$$
1+h(z)=e^{c}+h(z) e^{-c},
$$

where $c(=$ constant $)=\beta(z)-\alpha(z)$.

Clearly, if $h(z)=d$ (=constant, which cannot be zero!), then two cases may arise: (a) $\alpha^{\prime}(z)$ is a constant, and (b) $\alpha^{\prime}(z)=d \alpha^{\prime}(z+\tau), \alpha^{\prime} \not \equiv$ constant. 
Case (a) yields $h(z) \equiv 1$ and hence it follows from this and equation (14) that $e^{c}=1$, (i.e., $e^{\alpha}=e^{\beta}$ ), then, according to equation (7), $\alpha^{\prime}(z) \equiv 0$, giving a contradiction. Case (b) indicates that $\alpha^{\prime}(z)$ has an order at least 1 , which would lead to a contradiction, as before. Now if $h(z) \not \equiv$ constant, then from equation (14) we easily deduce that $e^{c}=1$, which is impossible, as we argued in case (a). Thus we have to conclude that $\alpha^{\prime}(z) \equiv 0$, and hence $\alpha(z) \equiv$ constant.

Now going back to equation (3) we consider

$$
f(z)=e^{\alpha} f(z+\tau),
$$

where $\alpha$ is a constant.

Let $b$ be any complex number such that $f(b+\tau) \neq 0$ and choose $a=\log [f(b) / f(b+\tau)] /-\tau$. Let us consider the expression

$$
f(z)=e^{a z} g(z),
$$

where $g(z)$ is an entire function. We are going to show that $g(z)$ is a periodic function with period $\tau$.

From equations (15) and (16) we obtain

$$
g(z) \equiv e^{\alpha+a \tau} g(z+\tau)
$$

which becomes

$$
g(z) \equiv g(z+\tau)
$$

using the assumed choice of $a$. This also completes the proof of the Theorem.

\section{REFERENCES}

1. W. K. Hayman, Meromorphic functions, Oxford Mathematical Monographs, Clarendon Press, Oxford, 1964. MR 29 \#1337.

2. R. Nevanlinna, Eindeutige analytische Funktionen, Die Grundlehren der Math. Wissenschaften, Band 46, Springer-Verlag, Berlin, 1936; English transl., Die Grundlehren der math. Wissenschaften, Band 162, Springer-Verlag, Berlin and New York, 1970. MR 43 \#5003.

Mathematics Research Center, Naval Research Laboratory 7840, Washington, D.C. 20390 